\title{
FAULT DETECTION FILTER FOR NONLINEAR SYSTEMS USING LINEAR APPROXIMATIONS
}

\author{
C. Navarro Hernandez ${ }^{*, 1}$ F.Crusca ${ }^{* *}$ M.Aldeen ${ }^{* * *}$ \\ S.P. Banks * \\ * Department of Automatic Control and Systems \\ Engineering, University of Sheffield, Mappin Street, \\ Sheffield S1 3JD, UK (c.l.navarro@shef.ac.uk) \\ ** Department of Electrical and Computer Systems \\ Engineering, Monash University, Clayton VIC 3800, \\ Australia \\ *** Department of Electrical and Electronic Engineering, \\ The University of Melbourne, Parkville VIC 3010, \\ Australia
}

\begin{abstract}
In this paper, the design of a fault detection filter for nonlinear systems is presented. The nonlinear system is represented as a sequence of linear timevarying approximations and at each linear approximation the design of an optimal stochastic fault detection filter for linear time-varying systems is applied. The final residual is primarily affected by a target fault and minimally by nuisance faults. Copyright (C)2005 IFAC
\end{abstract}

Keywords: Fault detection, Filter design, Linear equations, Nonlinear system, Time-varying systems

\section{INTRODUCTION}

The study of fault detection and isolation (FDI) methods for nonlinear systems has become of increasing importance in the last few years. Early FDI methods were focused on linear systems, however the fact that all physical systems are inherently nonlinear and that in many cases the system cannot be accurately described by a linear model made necessary the development of specific fault detection methods for nonlinear systems. Several approaches have been developed, some of the methods include nonlinear state transformations of the nonlinear system into a required form (Seliger et al., 1991) or the decomposition

\footnotetext{
1 Supported by CONACYT, Mexico and Monash University, Australia
}

of the nonlinear system into one part affected by the faults and another decoupled from them (Yang and Saif, 1996). Alcorta and Frank (1997) and Frank (1994) give a survey on observer-based approaches to FDI for nonlinear systems. However, the approaches usually consider a specific form of the nonlinear system, which limits the use of general models. On the other hand, there are well-known approaches in the study of FDI for linear time invariant systems, such as the residual generation approach and the unknown-input observer approach, which separates the faults into a target fault and several nuisance faults which are unobservable to the residual. There are also some attempts at studying fault detection methods for linear time-varying systems (LTV), such as (Edelmayer et al., 1997) based on a time-invariant representation of a LTI system corrupted by linear 
time-varying parametric perturbations and $(\mathrm{Xu}$ and Zhang, 2002) based on the design of an adaptive observer. Recently, a design which extends the unknown input observer to the time-varying case by designing a fault detection filter has been proposed (Chen et al., 2003). Using this idea, this paper deals with the design of a fault detection filter for nonlinear systems by combining the method of fault detection for LTV systems and an iteration technique in which the nonlinear dynamical system is represented as the limit of a sequence of linear time-varying approximations which converge to the solution of the nonlinear system under a local Lipschitz condition (TomasRodriguez and Banks, 2003). This technique allows the use of linear techniques without having to locally linearize the nonlinear system and has been previously applied in nonlinear control theory in optimal control (Cimen and Banks, 2004), and in the design of observers for nonlinear systems (Navarro Hernandez et al., 2003) among others.

The paper is organized as follows: Section 2 presents the principles of the linear approximation scheme, Section 3 outlines the design method of the fault detection filter for LTV systems. In Section 4 the techniques are combined to design a fault detection filter for nonlinear systems, Section 5 presents the convergence proof and Section 6 gives an example for a nonlinear system.

\section{APPROXIMATION SCHEME}

Consider a nonlinear control system written in the following form,

$$
\dot{x}=A(x) x+B(x) u, x(0)=x_{0} \in \mathbb{R}^{n}
$$

and introduce the sequence of linear time varying equations:

$$
\begin{aligned}
\dot{x}^{[0]}(t) & =A\left(x_{0}\right) x^{[0]}(t)+B\left(x_{0}\right) u^{[0]}(t) \\
\dot{x}^{[i]}(t) & =A\left(x^{[i-1]}(t)\right) x^{[i]}(t)+B\left(x^{[i-1]}(t)\right) u^{[i]}(t) \\
x^{[0]}(0) & =x_{0} \quad x^{[i]}(0)=x_{0}
\end{aligned}
$$

where $i=1,2, \ldots$ is the approximation number. If $A(x)$ and $B(x)$ are of appropriate dimension and locally Lipschitz, the solution of the sequence (2) converges to the solution of the original nonlinear system (1)in a compact time interval. Details can be found in Tomas-Rodriguez and Banks (2003). The convergence rate of the sequence of LTV systems to the nonlinear system depends on the system, initial conditions and the specific control problem. This subject is however still under study, as the current results on the rate of convergence are still quite restrictive.
Using this idea, a feedback control can be defined as $u^{[i]}(t)=-K\left(x^{[i-1]}(t)\right) x^{[i]}(t)$ and

$$
\begin{aligned}
& \dot{x}^{[i]}(t)= \\
& \left(A\left(x^{[i-1]}(t)\right)-B\left(x^{[i-1]}(t)\right) K\left(x^{[i-1]}(t)\right)\right) x^{[i]}(t)
\end{aligned}
$$

Then, by using the results in (Tomas-Rodriguez and Banks, 2003), the system (3) converges if $A(x), B(x), K(x)$ are locally Lipschitz and $u(t)=$ $-K(x) x(t)$ is a feedback control for the nonlinear system (1).

Remark 1. If the nonlinear system is stable, then the sequence of solutions (2) will converge to the nonlinear solution on any compact time interval $[0, T]$. By taking $T$ arbitrarily large and the iteration number large enough (depending on $T$ ), then that solution will be arbitrarily close to the equilibrium point.

\section{FAULT DETECTION FILTER FOR LTV SYSTEMS}

This section briefly presents the design of the fault detection filter for LTV systems proposed by Chen et al. (2003). This design extends the results of unknown input observers to the time varying case by solving an optimization problem. The objective of the filter is to detect a target fault while blocking nuisance faults in the presence of process and sensor noises. The filter places the nuisance faults into a minimal (C,A)-unobservability subspace for time-invariant systems and a similar invariant subspace for time-varying systems. The design is mainly based on maximizing the transmission of the target fault to the projected output error while minimizing the transmission from the nuisance faults by using a weighting factor and a projector; obtaining a residual which is primarily affected by the target faults. The filter can be robust with respect to disturbances.

Consider a linear time-varying, uniformly observable system,

$$
\begin{aligned}
\dot{x}(t) & =A x+B_{u} u+B_{w} w+F_{1} \mu_{1}+F_{2} \mu_{2} \\
y(t) & =C x+v
\end{aligned}
$$

where $u$ is the control input, $y$ is the measurement, $w$ is the process noise, $v$ is the sensor noise, $\mu_{1}$ is the target fault and $\mu_{2}$ is the nuisance fault containing all other faults to be blocked. $F_{1}$ is the target fault direction and $F_{2}$ is the nuisance fault direction. (Note that the dependance on time is not written explicitly).

The objective is to find a filter gain $L$ for the observer 


$$
\dot{\widehat{x}}=A \widehat{x}+B_{u} u+L(y-C \widehat{x})
$$

and a projector $\widehat{H}$ for the residual

$$
r=\widehat{H}(y-C \widehat{x})
$$

such that the residual is primarily affected by the target fault and minimally by the nuisance fault, process noise, sensor noise and initial condition error. The initial state $\left(x_{0}\right)$ is a random vector with variance $P_{0}$ and $\mu_{1}, \mu_{2}$ and $w$ are assumed to be zero mean, white noises with power spectral densities $Q_{1}, Q_{2}, Q_{w}$ and $V$, respectively, and are uncorrelated with each other and with $x\left(t_{0}\right)$.

The error is

$$
\begin{aligned}
e(t) & =\Phi\left(t, t_{0}\right) e\left(t_{0}\right) \\
& +\int_{t_{0}}^{t} \Phi(t, \tau)\left(F_{1} \mu_{1}+F_{2} \mu_{2}+B_{w} w-L v\right) d \tau
\end{aligned}
$$

subject to

$$
\frac{d}{d t} \Phi\left(t, t_{0}\right)=(A-L C) \Phi\left(t, t_{0}\right)
$$

where $\Phi\left(t_{0}, t_{0}\right)=I$ and the residual (4) is $r=$ $\widehat{H}(C e+v)$. Then, Chen et al. (2003) define a cost criterion based on the projected output error $r=\widehat{H} C e$ by first defining

$$
\begin{aligned}
h_{1}(t) & \triangleq \widehat{H} C \int_{t_{0}}^{t} \Phi(t, \tau) F_{1} \mu_{1} d \tau, \\
h_{2}(t) & \triangleq \widehat{H} C \int_{t_{0}}^{t} \Phi(t, \tau) F_{2} \mu_{2} d \tau, \\
h_{3}(t) & \triangleq \widehat{H} C\left[\Phi\left(t, t_{0}\right) e\left(t_{0}\right)\right. \\
& \left.+\int_{t_{0}}^{t} \Phi(t, \tau)\left(B_{w} w-L v\right) d \tau\right]
\end{aligned}
$$

The cost criterion then is

$$
\begin{aligned}
J= & \operatorname{tr}\left\{\frac{1}{\gamma} E\left[h_{2}(t) h_{2}(t)^{T}\right]+E\left[h_{3}(t) h_{3}(t)^{T}\right]\right. \\
& \left.-E\left[h_{1}(t) h_{1}(t)^{T}\right]\right\}
\end{aligned}
$$

where $E\left[h_{1}(t) h_{1}(t)^{T}\right]$ is the transmission from $\mu_{1}$ to $\widehat{H} C e, E\left[h_{2}(t) h_{2}(t)^{T}\right]$ the transmission from $\mu_{2}$ to $\widehat{H} C e, E\left[h_{3}(t) h_{3}(t)^{T}\right]$ the transmission from $w, v$ and $e\left(t_{0}\right)$ to $\widehat{H} C e$, and $E[\bullet]$ is the expectation operator. $e\left(t_{0}\right)$ is a zero mean random vector with variance $P_{0}$ if $\widehat{x}\left(t_{0}\right)=E\left[x\left(t_{0}\right)\right]$. The objective is to find a filter gain $L$ and projector $\widehat{H}$ which minimize (5). The positive scalar $\gamma$ is used as a weight on the nuisance fault. The transmission of the faults is also affected by the power spectral densities $Q_{1}$ and $Q_{2}$ which are design parameters and by $Q_{w}, V$ and $P_{0}$ which can have physical values. The filter is robust with respect to the process and sensor noises and recovers the geometric structure of the unknown input observer in the limit as $\gamma \rightarrow 0$. When it is not at the limit, the filter is an approximate unknown input observer and the nuisance fault is partially blocked, see (Chen et al., 2003) for details and proofs.

The minimization problem can be rewritten as

$$
\begin{aligned}
& \min _{L, \widehat{H}} \operatorname{tr}\left[\widehat{H} C \int_{t_{0}}^{t} \Phi(t, \tau)\left(L-P C^{T} V^{-1}\right) V\right. \\
& \left.\left(L-P C^{T} V^{-1}\right)^{T} \Phi(t, \tau)^{T} d \tau C^{T} \widehat{H}+\widehat{H} C P(t) C^{T} \widehat{H}\right]
\end{aligned}
$$

subject to (3) and that $\widehat{H}$ is a projector, where

$$
\begin{gathered}
\dot{P}=A P+P A^{T}-P C^{T} V^{-1} C P+\frac{1}{\gamma} F_{2} Q_{2} F_{2}^{T} \\
-F_{1} Q_{1} F_{1}^{T}+B_{w} Q_{w} B_{w}^{T}, \quad P\left(t_{0}\right)=P_{o}
\end{gathered}
$$

From (7), the optimal filter gain is

$$
L=P C^{T} V^{-1}
$$

By applying (6) to (8) and substituting $\widehat{H}=\rho \rho^{T}$, the minimization problem (6) reduces to

$$
\min _{\rho} \operatorname{tr}\left[\rho^{T} C P(t) C^{T} \rho\right]
$$

subject to $\rho^{T} \rho=I$. By adjoining the constraint to the cost criterion, the first order necessary condition is

$$
C P(t) C^{T} \rho=\rho \lambda
$$

and the solution of the optimal $\rho$ depends on the rank of $\widehat{H}$. If the projector is of rank one, then the optimal projector is

$$
\widehat{H}^{*}=\rho_{m} \rho_{m}^{T}
$$

where $\lambda_{1} \geq \lambda_{2} \geq \ldots \geq \lambda_{m}$ are the eigenvalues of $C P(t) C^{T}$ and $\rho_{1}, \rho_{2}, \ldots, \rho_{m}$ the associated eigenvectors. The rank of $\widehat{H}$ can also be chosen as $m-p_{2}$ where $p_{2}=d i m F_{2}$ are the number of large eigenvalues near the limit when $\gamma$ is small. Then the optimal projector is:

$$
\widehat{H}^{*}=\left[\rho_{m} \rho_{m-1} \ldots \rho_{p_{2}+1}\right]\left[\rho_{m} \rho_{m-1} \ldots \rho_{p_{2}+1}\right]^{T}
$$

\section{FAULT DETECTION FILTER FOR NONLINEAR SYSTEMS}

In this section, the algorithm for the nonlinear fault detection filter is presented, by combining 
the design proposed by Chen et al. (2003) and the approximation scheme for nonlinear systems presented in (Tomas-Rodriguez and Banks, 2003). Consider the following observable nonlinear system subject to a target fault $\mu_{1}$ and a nuisance fault $\mu_{2}$ which are unknown and arbitrary functions of time.

$$
\begin{aligned}
\dot{x}(t) & =A(x) x(t)+B(x) u(t)+F_{1}(x) \mu_{1}(t) \\
& +F_{2}(x) \mu_{2}(t) ; x(0)=x_{0} \\
y(t) & =C(x) x(t)
\end{aligned}
$$

and introduce the approximation scheme of Section 2. For the first approximation,

$$
\begin{aligned}
\dot{x}^{[0]}(t) & =A\left(x_{0}\right) x^{[0]}(t)+B\left(x_{0}\right) u^{[0]}(t)+F_{1}\left(x_{0}\right) \mu_{1}(t) \\
& +F_{2}\left(x_{0}\right) \mu_{2}(t) \\
y^{[0]}(t) & =C\left(x_{0}\right) x^{[0]}(t) ; x^{[0]}(0)=x_{0}
\end{aligned}
$$

and for $i \geq 1$,

$$
\begin{aligned}
\dot{x}^{[i]}(t) & =A\left(x^{[i-1]}(t)\right) x^{[i]}(t)+B\left(x^{[i-1]}(t)\right) u^{[i]}(t) \\
& +F_{1}\left(x^{[i-1]}(t)\right) \mu_{1}(t)+F_{2}\left(x^{[i-1]}(t)\right) \mu_{2}(t) \\
y^{[i]}(t) & =C\left(x^{[i-1]}(t)\right) x^{[i]}(t) ; x^{[i]}(0)=x_{0}
\end{aligned}
$$

Hence, the following sequence of linear observers can be defined,

$$
\begin{aligned}
\dot{\bar{x}}^{[0]}(t) & =A\left(x_{0}\right) \widehat{x}^{[0]}(t)+B\left(x_{0}\right) u^{[0]}(t) \\
& +L^{[0]}(t)\left(y^{[0]}(t)-C\left(x_{0}\right) \widehat{x}^{[0]}(t)\right) ; \\
\widehat{x}^{[0]}(0) & =\widehat{x}_{0}
\end{aligned}
$$

and for $i \geq 1$,

$$
\begin{aligned}
\dot{\hat{\bar{x}}}^{[i]}(t) & =A\left(x^{[i-1]}(t)\right) \widehat{x}^{[i]}(t)+B\left(x^{[i-1]}(t)\right) u^{[i]}(t) \\
& +L^{[i]}(t)\left(y^{[i]}(t)-C\left(x^{[i-1]}(t)\right) \widehat{x}^{[i]}(t)\right) ; \\
\widehat{x}^{[i]}(0) & =\widehat{x}_{0}
\end{aligned}
$$

with residual

$$
r^{[0]}(t)=\widehat{H}^{[0]}(t)\left(y^{[0]}(t)-C\left(x_{0}\right) \widehat{x}^{[0]}(t)\right)
$$

and for $i \geq 1$,

$$
r^{[i]}(t)=\widehat{H}^{[i]}(t)\left(y^{[i]}(t)-C\left(x^{[i-1]}(t)\right) \widehat{x}^{[i]}(t)\right)
$$

At each approximation, a filter is found, obtaining then a sequence of fault detection filters which will finally give a filter for the original nonlinear system (9). To obtain the values of $L$ and $H$ at each approximation, the design proposed by Chen et al. (2003) is used, therefore, it is necessary to solve a
Ricatti equation at each LTV approximation. This means that the solution will be suboptimal for the original nonlinear system, in order to obtain an optimal solution it is necessary to use HamiltonJacobi methods as explained in Cimen and Banks (2004). Despite this, the final filter will still be a fault detector for the nonlinear system.

Using the ideas from Section 3, for the first approximation it is necessary so solve

$$
\begin{aligned}
\dot{P}^{[0]}(t) & =A\left(x_{0}\right) P^{[0]}(t)+P^{[0]}(t) A\left(x_{0}\right)^{T} \\
& -P^{[0]}(t) C\left(x_{0}\right)^{T} V^{-1} P^{[0]}(t) \\
& +\frac{1}{\gamma} F_{2}\left(x_{0}\right) Q_{2} F_{2}\left(x_{0}\right)^{T}-F_{1}\left(x_{0}\right) Q_{1} F_{1}\left(x_{0}\right)^{T}
\end{aligned}
$$

and for $i \geq 1$

$$
\begin{aligned}
\dot{P}^{[i]}(t) & =A\left(\left(x^{[i-1]}(t)\right) P^{[i]}(t)+P^{[i]}(t) A\left(\left(x^{[i-1]}(t)\right)^{T}\right.\right. \\
& -P^{[i]}(t) C\left(x^{[i-1]}(t)\right)^{T} V^{-1} P^{[i]}(t) \\
& +\frac{1}{\gamma} F_{2}\left(x^{[i-1]}(t)\right) Q_{2} F_{2}\left(x^{[i-1]}(t)\right)^{T} \\
& -F_{1}\left(x^{[i-1]}(t)\right) Q_{1} F_{1}\left(x^{[i-1]}(t)\right)^{T}, P^{[i]}(0)=P_{0}
\end{aligned}
$$

obtaining then, the following sequence of filter gains. For the first approximation

$$
\begin{aligned}
& L^{[0]}(t)=P^{[0]}(t) C\left(x_{0}\right)^{T} V^{-1} ; \\
& L^{[0]}(0)=P_{0} C\left(x_{0}\right)^{T} V^{-1}
\end{aligned}
$$

and for $i \geq 1$

$$
\begin{aligned}
& L^{[i]}(t)=P^{[i]}(t) C\left(x^{[i-1]}(t)\right)^{T} V^{-1} ; \\
& L^{[i]}(0)=P_{0} C\left(x_{0}\right)^{T} V^{-1}
\end{aligned}
$$

Now, to obtain the projector, consider the following sequence

$$
C\left(x_{0}\right) P^{[0]}(t) C\left(x_{0}\right)^{T} \rho=\rho \lambda
$$

where $\lambda_{1}^{[0]}(t) \geq \lambda_{2}^{[0]}(t) \geq \ldots \geq \lambda_{m}^{[0]}(t)$ are the eigenvalues of $\bar{C}\left(x_{0}\right) P^{[0]}(t) C\left(x_{0}\right)^{T}$ and the associated eigenvectors are $\rho_{1}^{[0]}(t), \rho_{2}^{[0]}(t), \ldots, \rho_{m}^{[0]}(t)$. An optimal projector of rank 1 for the first approximation is

$$
H^{[0] *}(t)=\rho_{m}^{[0]}(t)\left(\rho_{m}^{[0]}(t)\right)^{T}
$$

and for $i \geq 1$

$\lambda_{1}^{[i]}(t) \geq \lambda_{2}^{[i]}(t) \geq \ldots \geq \lambda_{m}^{[i]}(t)$ are the eigenvalues of $C\left(x^{[i-1]}(t)\right) P^{[i]}(t) C\left(x^{[i-1]}(t)\right)^{T}$ and the associated eigenvectors are $\rho_{1}^{[i]}(t), \rho_{2}^{[i]}(t), \ldots, \rho_{m}^{[i]}(t)$. The projector of rank 1 for the approximations $i \geq 1$ is defined as 


$$
H^{[i] *}(t)=\rho_{m}^{[i]}(t) \rho_{m}^{[i]}(t)^{T}
$$

\section{CONVERGENCE PROOF}

Consider the following nonlinear system subject to a fault $\mu_{1}(t)$ with direction $F$ :

$\dot{x}(t)=A(x) x(t)+B(x) u(t)+F(x) \mu_{1}(t) ; x(0)=x_{0}$

$y(t)=C(x) x(t)$

this can be rewritten as

$$
\begin{aligned}
\dot{x}(t) & =A(x) x(t)+G(x) g(t) ; x(0)=x_{0} \\
y(t) & =C(x) x(t)
\end{aligned}
$$

where

$$
G(x)=[B(x) F(x)] \quad g(t)=\left[\begin{array}{c}
u(t) \\
\mu_{1}(t)
\end{array}\right]
$$

then, from the results in (Tomas-Rodriguez and Banks, 2003), the sequence of approximations $\left\{x^{[i]}(t)\right\}$ is a Cauchy sequence that will converge uniformly on $[0, \tau]$ for $\tau \geq 0$ in the space $C\left([0, \tau], \mathbb{R}^{n}\right)$ to the real solution of the nonlinear system (12) if $A(x), G(x), C(x)$ are locally Lipschitz.

In the same way, the sequence of approximations $\left\{\widehat{x}^{[i]}(t)\right\}$ is also a Cauchy sequence; since the matrices $L^{[i]}(t)$ of the linear observer approximations depend on the matrices $A\left(x^{[i-1]}\right), G\left(x^{[i-1]}\right)$ and $C\left(x^{[i-1]}\right)$, which are locally Lipschitz in $x^{[i-1]}$. The proof that the proposed filter design for LTV systems behaves in the limit as an unknown input observer is found in (Chen et al., 2003) and (Chung and Speyer, 1999). Then, at each approximation there exists a residual which is more sensitive to the target fault, defined as

$$
r^{[i]}(t)=\widehat{H}^{[i]}(t) C\left(x^{[i-1]}(t)\right)\left(x^{[i]}(t)-\hat{x}^{[i]}(t)\right)
$$

where $\left\{x^{[i]}(t)\right\}$ and $\left\{\widehat{x}^{[i]}(t)\right\}$ are Cauchy sequences, and $\left\|x(t)-x^{[i]}(t)\right\|$ and $\left\|\widehat{x}(t)-\widehat{x}^{[i]}(t)\right\|$ are bounded. Then, at the final approximation as $\left\{x^{[i]}(t)\right\}$ converges to the real states, and $\left\{\widehat{x}^{[i]}(t)\right\}$ to the estimates of the nonlinear system, a filter for the nonlinear system is achieved with a residual $r^{[i]}(t)$ which maximizes the effect of the target fault and minimizes the effect of the nuisance fault.

\section{EXAMPLE}

Consider the nonlinear system
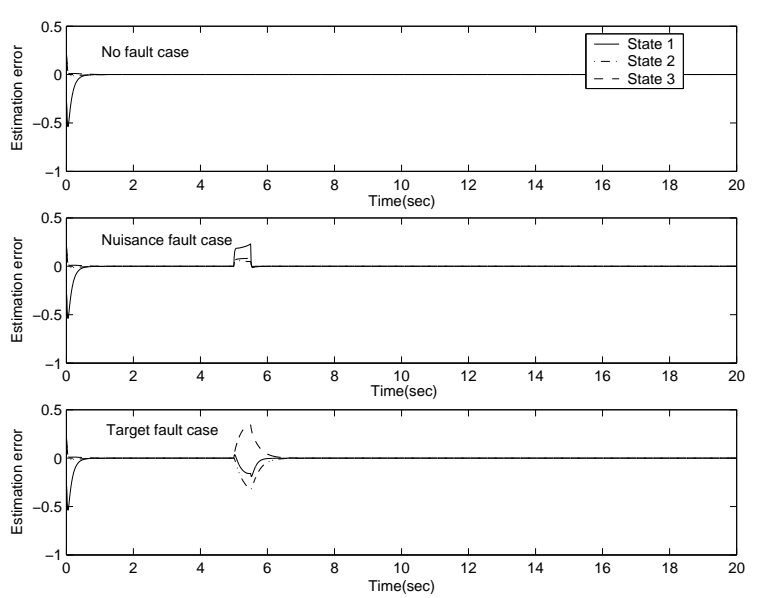

Fig. 1. Estimation error

$$
\begin{aligned}
A & =\left(\begin{array}{ccc}
-8 & x_{2} & 0 \\
x_{3} & -5 & x_{1} \\
-x_{2} & x_{3} & -x_{2}
\end{array}\right), C=\left(\begin{array}{lll}
0 & 1 & 0 \\
0 & 0 & 1
\end{array}\right) \\
F_{1} & =\left(\begin{array}{l}
1 \\
0 \\
1
\end{array}\right), F_{2}=\left(\begin{array}{c}
5-2 \cos \left(x_{1}\right) \\
1 \\
1+\sin \left(x_{2}\right)
\end{array}\right)
\end{aligned}
$$

where $F_{1}$ is the target fault direction and $F_{2}$ the nuisance fault direction. The filter is solved with $Q_{1}=1, Q_{2}=1, V=I$ and $P_{0}=I$ for $t \in[0,20]$. There are no process and sensor noises. The faults are pulses of magnitude 3 that occur from $t=5 \mathrm{sec}$ to $\mathrm{t}=5.5 \mathrm{sec}$. The initial conditions of the state and estimates are $x_{0}=[.2, .2, .4]$ and $\hat{x_{0}}=[.5,0, .2]$ respectively and the weighting factor is $\gamma=10^{-4}$.

The nonlinear system was represented as a sequence of LTV equations of the form $(10,11)$ and a fault detection filter was designed for each LTV system. The number of iterations in this example was 25 . Figures 1 and 2 show the estimation error and residual respectively subject to different fault cases at the final approximation. As expected, the residual is primarily affected by the target fault. Figure 3, shows the norm of the residual due to the nuisance fault at different approximations. As the number of approximations is increased, it is ensured that the residual is a result of the faults in the nonlinear system, as the solution of the approximations converges to the solution of the nonlinear problem. Although the above example does not come from a practical situation, this technique can be applied to nonlinear systems such as the bioreactor studied before by Gauthier et al. (1992) and Xu and Zhang (2004) and other systems with practical interpretations. 

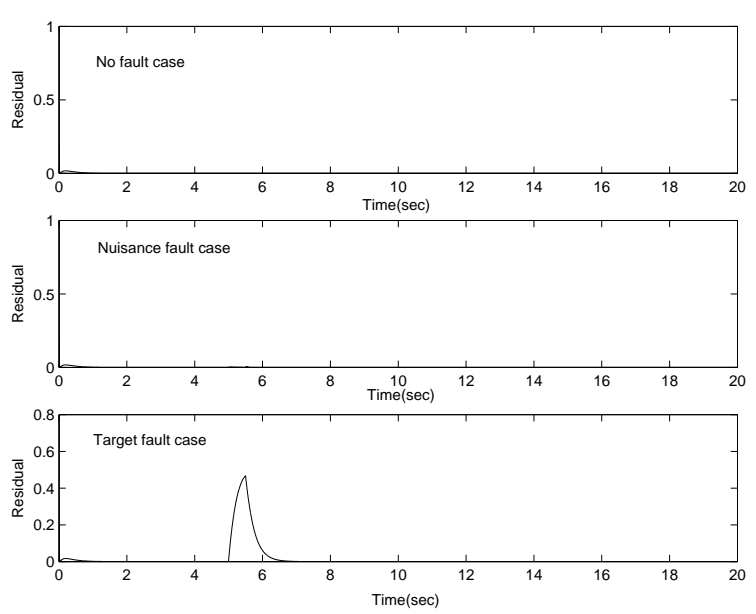

Fig. 2. Time response of the residual

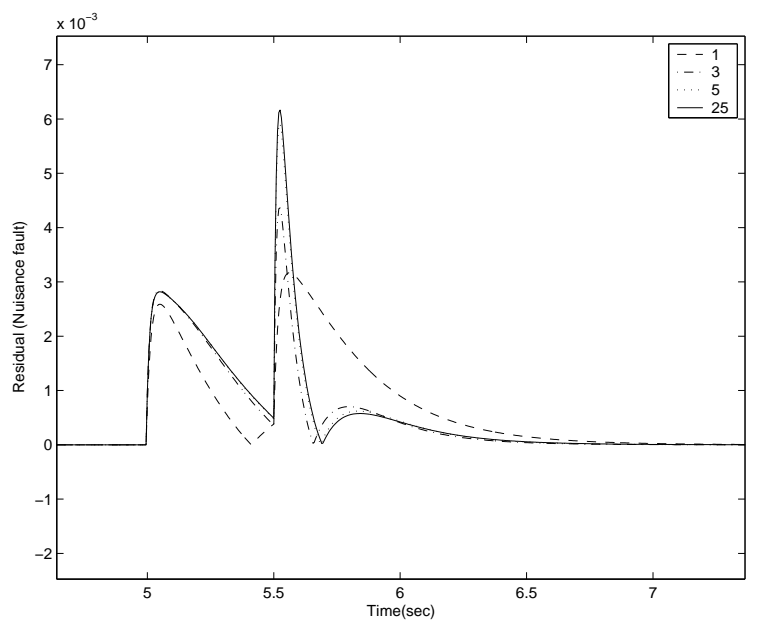

Fig. 3. Residual at different approximations (Nuisance fault)

\section{CONCLUSIONS}

This paper introduces a new technique to design a fault detection filter for nonlinear systems based on the replacement of the nonlinear system by a sequence of linear time-varying approximations and the design of an optimal stochastic fault detection filter for LTV systems which approximates the unknown input observer in the limit. The condition required here is very mild, i.e. a local Lipschitz condition, which is necessary even for uniqueness of solutions of differential equations and therefore is satisfied in many engineering problems. The authors are also exploring methods to be able to apply this technique even if the system does not satisfy the Lipschitz condition. Once the sequence of LTV systems is obtained, the filters at each approximation are designed solving a minimization problem and the filter at the final approximation represents a filter for the nonlinear system. The example shows that the norm of the residual obtained from the design was primarily affected by the target fault and minimally by the nuisance one.

\section{REFERENCES}

Alcorta Garcia,E. and Frank, PM. (1997). Deterministic nonlinear observer-based approaches to fault diagnosis: a survey Control Engineering Practice, 5, 663-670.

Chen,R., Mingori,D. and Speyer, J. (2003). Optimal stochastic fault detection filter, Automatica, 39, 377-390.

Chung,W., and Speyer, J. (1998). A Game Theoretic Fault Detection Filter, IEEE Transactions on Automatic Control, 42, No. 43. 143-161.

Cimen, T. and Banks, S.P. (2004). Global optimal feedback control for general nonlinear systems with nonquadratic performance criteria, Systems and Control Letters, 53, Issue 5, 327-346.

Frank,P.M., (1994). On-line fault detection in uncertain nonlinear systems using diagnostic observers: a survey,Int. J. Systems Sci., 25, 2129-2154.

Edelmayer,A., Bokor,J., Szigeti,F. and Keviczky, L. (1997). Robust Detection Filter in the Presence of Time-Varying System Perturbations, Automatica, 33, No. 3, 471-475.

Gauthier,J.P., Hammouri,H. and Othman,S. (1992). A simple Observer for Nonlinear Systems Applications to Bioreactors, IEEE. Trans. Automatic Control, 37, No. 6, 874-879.

Navarro Hernandez, C.; Banks, S.P. and Aldeen, M. (2003) Observer design for nonlinear systems using linear approximations, IMA J. Math Cont and Inf 20, 359-370.

Seliger, R. and Frank, P.M. (1991) FaultDiagnosis by disturbance decoupled nonlinear observers Proc. of the 30th IEEE Conf. on Decision and Control, Brighton, England.

Tomas-Rodriguez, M. and Banks, S.P. (2003), Linear approximations to Nonlinear Dynamical Systems with Applications to Stability and Spectral Theory, IMA J. Math Cont and Inf, 20, 89-103.

Yang, H. and Saif, SM. (1996) Monitoring and diagnostics of a class of nonlinear system using a nonlinear unknown input observer Proc. of the 1996 IEEE Int. Conf. on Control Applications, Dearborn, MI.

Xu,A., Zhang, Q. (2002) Fault Detection and Isolation based on Adaptive Observers for Linear Time Varying Systems , 15th Triennial World Congress, Barcelona, Spain.

Xu,A., Zhang, Q. (2004) Nonlinear system fault diagnosis based on adaptive estimation, Automatica, 40, 1181-1193. 